\title{
O volume de fluxo e a velocidade de pico sistólico ao ultrassom vascular com Doppler intraoperatório como preditores de perviedade precoce na fístula arteriovenosa autógena para hemodiálise
}

\author{
Intraoperative vascular Doppler ultrasound blood flow and peak systolic velocity \\ predict early patency in hemodialysis arteriovenous fistula \\ Guilherme de Castro-Santos' (D), Gabriella Yuka Shiomatsu¹ (D), Rafaela Martins dos Santos Oliveira' (D), \\ Ricardo Jayme Procópio ${ }^{1}$ (D), Túlio Pinho Navarro ${ }^{1 D}$
}

\begin{abstract}
Resumo
Contexto: A insuficiência renal crônica é um problema de saúde pública mundial. A hemodiálise é a principal terapia renal substitutiva. As fístulas arteriovenosas (FAV) são uma possível escolha, mas apresentam altas taxas de falência. Objetivos: Este estudo tem como objetivo avaliar a relação entre as variáveis hemodinâmicas ao ultrassom vascular com Doppler no intraoperatório e a perviedade precoce da FAV para hemodiálise. Métodos: Tratou-se de um estudo prospectivo observacional. Os pacientes consecutivos foram submetidos a FAV com ultrassonografia vascular com Doppler em intraoperatório nos dias 1, 7, 30 e 60. Eles foram divididos em grupos quanto à presença ou não de perviedade primária e secundária, e o volume de fluxo (VF) e a velocidade de pico sistólico (VPS) foram comparados. Foram realizadas curvas receiver operating characteristic (ROC), com definição de valores de VPS e VF com sensibilidade (S) e especificidade (E). Resultados: Foram analisados 47 pacientes, os quais preencheram os critérios de inclusão. Os valores de VPS e VF intraoperatório foram maiores nos pacientes com perviedade primária e secundária comparados àqueles com falência. Os seguintes valores apresentaram maiores sensibilidade e especificidade para predizer perviedade primária aos 30 dias: $106 \mathrm{~cm} / \mathrm{s}$ para VPS venoso, S: 75\%, E: 71,4\%; e 290,5 mL/min para VF arterial, S: 80,6\%, E: 85,7\%. Para perviedade secundária aos 30 dias, foram observados: $106 \mathrm{~cm} / \mathrm{s}$ para VPS arterial, S: 72,7\%, E: 100\%; e $230 \mathrm{~mL} / \mathrm{min}$ para VF venoso, com S: 86,4\%, E: 100\%. Para a perviedade primária no $60^{\circ}$ dia, foram observados: $106 \mathrm{~cm} / \mathrm{s}$ para VPS venoso, S: 74,4\%, E: 62,5\%; e 290,5 mL/min para VF arterial, S: 80\%, E: 75\%. Conclusões: A velocidade de pico sistólico e o VF ao ultrassom vascular com Doppler intraoperatório são preditores de perviedade precoce na FAV para hemodiálise.
\end{abstract}

Palavras-chave: fístula arteriovenosa; perviedade; ultrassom intraoperatório; volume de fluxo; velocidade sistólica de pico.

\begin{abstract}
Background: Chronic kidney disease is a major public health problem. Hemodialysis is the most common renal replacement therapy. Arteriovenous fistulas (AVF) are a possible access option, but early failure rates remain high. Objectives: to investigate the value of intraoperative vascular Doppler ultrasound for predicting early AVF patency. Methods: Prospective observational study. Consecutive patients undergoing AVF were assessed with vascular Doppler ultrasonography intraoperatively and on days 1, 7, 30, and 60. Patients were divided into groups according to presence or absence of primary and secondary patency. Blood flow (BF) and peak systolic velocity (PSV) were compared. ROC curves were plotted and used to define the PSV and BF values that yielded greatest sensitivity (Sens) and specificity (Spec). Results: 47 patients met the inclusion criteria and were analyzed. Higher intraoperative PSV and BF values were observed in patients who had primary and secondary patency than in patients with access failure. The values with greatest sensitivity and specificity for predicting 30-day primary patency were $106 \mathrm{~cm} / \mathrm{s}$ for venous PSV (Sens: $75 \%$ and Spec: $71.4 \%$ ) and $290.5 \mathrm{ml} / \mathrm{min}$ for arterial blood flow (Sens: $80.6 \%$ and Spec $85.7 \%$ ). Values for 30-day secondary patency were $106 \mathrm{~cm} / \mathrm{s}$ for arterial PSV (Sens: $72.7 \%$, Spec: 100\%) and $230 \mathrm{ml} / \mathrm{min}$ for venous blood flow (Sens: 86.4\%, Spec100\%). Values for 60-day primary patency were $106 \mathrm{~cm} / \mathrm{s}$ for venous PSV (Sens: $74.4 \%$, Spec: 62.5\%) and 290.5 ml/min for arterial blood flow (Sens: 80\%, Spec: 75\%). Conclusions: Peak systolic velocity and blood flow measured using intraoperative vascular Doppler ultrasound can predict early patency of hemodialysis arteriovenous fistulas.
\end{abstract}

Keywords: arteriovenous fistula; patency; intraoperative ultrasound; blood flow; peak systolic velocity.

Como citar: Castro-Santos G, Shiomatsu GY, Oliveira RMS, Procópio RJ, Navarro TP. O volume de fluxo e a velocidade de pico sistólico ao ultrassom vascular com Doppler intraoperatório como preditores de perviedade precoce na fístula arteriovenosa autógena para hemodiálise. J Vasc Bras. 2021;20:e20210098. https://doi.org/10.1590/1677-5449.210098

${ }^{1}$ Universidade Federal de Minas Gerais - UFMG, Belo Horizonte, MG, Brasil.

Fonte de financiamento: Nenhuma.

Conflito de interesse: Os autores declararam não haver conflitos de interesse que precisam ser informados.

Submetido em: Junho 07, 2021. Aceito em: Setembro 30, 2021.

O estudo foi realizado no Hospital das Clínicas da Universidade Federal de Minas Gerais (UFMG), Belo Horizonte, MG, Brasil.

Copyright $(\mathrm{C} 2021$ Os autores. Este é um artigo publicado em acesso aberto (Open Access) sob a licença Creative Commons Attribution, que permite uso, distribuição e reprodução em qualquer meio, sem restrições desde que o trabalho original seja corretamente citado. 


\section{INTRODUÇÃO}

A doença renal crônica (DRC) é um problema de saúde pública mundial ${ }^{1}$. Em 2017, estimou-se 697,5 milhões de casos de DRC na população global, sendo 16,7 milhões apenas no Brasil ${ }^{1,2}$. A principal modalidade de terapia renal substitutiva é a hemodiálise. Projeta-se que a demanda pela hemodiálise aumente em mais do que o dobro entre 2010 e $2030^{3}$.

Entre os diferentes tipos de acesso para hemodiálise, há preferência pela fístula arteriovenosa autógena $(\mathrm{FAV})^{4}$. Se comparada às próteses e aos cateteres, a FAV possui maior perviedade a longo prazo e menores taxas de complicações, infecções e mortalidade ${ }^{5,6}$. Entretanto, sabe-se que as taxas de perviedade não são ideais, e a falência precoce é um fator de grande impacto, atingindo cerca de $20 \%$ das $\mathrm{FAVs}^{7,8}$.

A ultrassonografia vascular com Doppler intraoperatória mostra-se como ferramenta de grande importância, uma vez que torna possível acompanhar variáveis hemodinâmicas nos pacientes submetidos à confecção da $\mathrm{FAV}^{9,10}$. Este estudo teve como objetivo avaliar a relação entre as variáveis hemodinâmicas ao ultrassom vascular com Doppler no intraoperatório e a perviedade precoce da FAV para hemodiálise.

\section{MÉTODOS}

$\mathrm{O}$ estudo foi autorizado pelo comitê de ética da Universidade Federal de Minas Gerais (UFMG), Belo Horizonte, Minas Gerais (MG), Brasil, CAAE: 03241718.6.0000.5149, número do parecer: 3.257.774, registrado no Registro Brasileiro de Ensaios Clínicos (Rebec), número do UTN: U1111-1247-880. Todos os pacientes assinaram o Termo de Consentimento Livre e Esclarecido, e a confidencialidade dos dados dos participantes foi plenamente respeitada em todos os processos.

Tratou-se de um estudo observacional do tipo coorte prospectivo. Foram selecionados pacientes consecutivos, maiores de 18 anos submetidos à confecção eletiva de FAV para hemodiálise no Hospital das Clínicas da UFMG, Belo Horizonte, MG, Brasil, entre maio de 2019 e dezembro de 2020. Pacientes submetidos à confecção do acesso com prótese arteriovenosa foram excluídos. Para reduzir o risco de viés de seleção, todos os pacientes submetidos à confecção de acesso definitivo para hemodiálise no período estudado foram convidados a participar do estudo. Todos os pacientes convidados aceitaram a participação.

A avaliação pré-operatória incluiu exame clínico e mapeamento ao ultrassom vascular com Doppler. Foi dada preferência para a anastomose arteriovenosa o mais distal possível, com diâmetro mínimo para a artéria doadora de $2 \mathrm{~mm}$ e para a veia receptora de $3 \mathrm{~mm}$. Durante o intraoperatório, foi realizado exame de ultrassonografia vascular com Doppler. As fístulas e os exames de ultrassonografia vascular com Doppler foram realizados de acordo com os protocolos do hospital e pelo mesmo cirurgião vascular.

\section{Procedimento cirúrgico}

As fístulas radiocefálica, braquiocefálica, braquiobasílica e ulnar-basílica foram confeccionadas de acordo com os protocolos do hospital, com bloqueio do plexo braquial ou anestesia local e administração local intra-arterial e intravenosa de solução de heparina na proporção de 1:100. As fístulas braquiobasílicas foram confeccionadas em uma única intervenção ${ }^{11} \mathrm{com}$ deslocamento superficial e anterior da veia. As fístulas braquiobraquiais foram realizadas em tempo único ou em dois tempos. A cirurgia em tempo único foi realizada utilizando a técnica descrita por Bazan e Schanzer ${ }^{12}$. A cirurgia em dois tempos foi realizada com anastomose laterolateral entre os vasos braquiais com posterior superficialização da veia mais adequada conforme os critérios de maturação.

\section{Avaliação com ultrassonografia vascular com Doppler}

A ultrassonografia vascular com Doppler foi realizada usando os aparelhos Philips CX 50 US (Philips Medical Systems, Andover, MA, EUA) e um transdutor linear L9-3 (frequência 9-3 MHz), para os exames intraoperatórios, e Toshiba Aplio 300 US (Toshiba Medical Systems, Tóquio, Japão) e um transdutor de matriz linear L9-3 (frequência 9-3 MHz) para os exames pós-operatórios. Dessa forma, não houve comparação direta de variáveis entre aparelhos diferentes.

Para o exame intraoperatório, considerou-se os valores obtidos no momento imediatamente após a anastomose arteriovenosa. Os diâmetros da artéria e da veia-alvo foram medidos como a distância vertical entre as paredes externas da artéria e da veia, usando o paquímetro eletrônico na máquina de ultrassonografia vascular com Doppler. Os parâmetros hemodinâmicos foram realizados com uma varredura sagital da artéria e da veia-alvo, a aproximadamente $3 \mathrm{~cm}$ proximalmente à anastomose, e a imagem de ultrassom modo $\mathrm{B}$ foi otimizada. O modo de ultrassom Doppler de onda pulsada foi, então, ativado, o gate size foi localizado no centro do lúmen arterial ou venoso e o tamanho da amostra foi ajustado para $3 \mathrm{~mm}$. Em seguida, o ângulo de insonação, definido como o ângulo entre o feixe de ultrassom e a direção do fluxo sanguíneo, foi ajustado e mantido em 60 graus ou menos. $\mathrm{O}$ traçado da forma de onda espectral Doppler de onda pulsada foi, então, ativado, e a escala, ajustada. Uma vez que uma forma de onda espectral Doppler de onda pulsada ideal foi alcançada, ela foi automaticamente traçada, e os parâmetros hemodinâmicos vasculares foram exibidos e registrados. Os parâmetros incluíram velocidade sistólica de pico (VPS, $\mathrm{cm} / \mathrm{s}$ ) e velocidade 
média $(\mathrm{Vm}, \mathrm{cm} / \mathrm{s})$. Os valores exibidos eram de uma média de três ciclos cardíacos consecutivos. A área da seção transversal do vaso foi calculada assumindo que a artéria e a veia tinham uma seção transversal circular. O volume de fluxo (VF) foi subsequentemente calculado como um produto da Vm e da área transversal, utilizando o software da máquina de ultrassom vascular com Doppler.

\section{Avaliação da perviedade}

A perviedade foi considerada como a presença de fluxo intravascular em veia receptora e avaliada através do ultrassom vascular com Doppler em intraoperatório e nos dias 1, 7, 30 e 60 do pós-operatório. A perviedade primária foi considerada como fístulas pérvias sem nenhum tipo de intervenção. A perviedade secundária foi considerada como fístulas pérvias com e sem intervenções.

\section{Cálculo do tamanho da amostra}

Foram utilizadas medidas assimétricas de amostragem entre os grupos (4:1), considerando a perviedade no D30 vs. o VF da artéria, com alfa 0,05 e beta 0,2 , e, utilizando os dados de Saucy et al. ${ }^{13}$, em que média $1=230$ (194) e média $2=98$ (65), haveria suficiência para $25+6$ pacientes, contemplando a amostra deste estudo.

\section{Análise estatística}

Os pacientes foram divididos em grupos quanto à presença ou não de perviedade primária e secundária nos dias 1, 7, 30 e 60 após a cirurgia. Variáveis individuais, demográficas e hemodinâmicas ao ultrassom vascular com Doppler em intraoperatório - VPS e VF arteriais e venosos - foram comparadas entre os grupos. Para avaliação da normalidade, foi utilizado o teste de Shapiro-Wilk. A comparação entre grupos foi feita por meio dos testes $t$ de Welch, de Mann-Whitney e exato de Fisher, quando adequados, e os modelos de previsão de perviedade foram analisados por meio da curva receiver operating characteristic (ROC). As taxas de perviedade foram demonstradas por meio de curvas de Kaplan-Meier. Todas as análises foram realizadas no software Prism GraphPad 9.0.0 (GraphPad Software, San Diego, CA, EUA) para iOS.

\section{RESULTADOS}

Cinquenta e um pacientes foram selecionados para o estudo. Destes, quatro foram submetidos à confecção do acesso por meio de prótese arteriovenosa de politetrafluoroetileno (PTFE) e excluídos da amostra (Figura 1). Os dados demográficos e individuais estão demonstrados nas Tabelas 1 e 2. Não houve perda de participantes no período.

As taxas de perviedade primária e secundária foram, respectivamente, $89,4 \%$ e $93,6 \%$ no dia 1 ; $87,2 \%$ e $93,6 \%$ no dia $7 ; 86,96 \%$ e $96,62 \%$ no 30 ; e $82,98 \%$ e $91,49 \%$ no dia 60 do pós-operatório (Figura 2). Dos cinco pacientes que trombosaram no dia 1 do pós-operatório, dois foram submetidos a tromboembolectomia com cateter de Fogarty, seguido por reconfecção da anastomose e anticoagulação sistêmica com heparina não fracionada e varfarina; um paciente foi submetido a confecção de nova anastomose mais

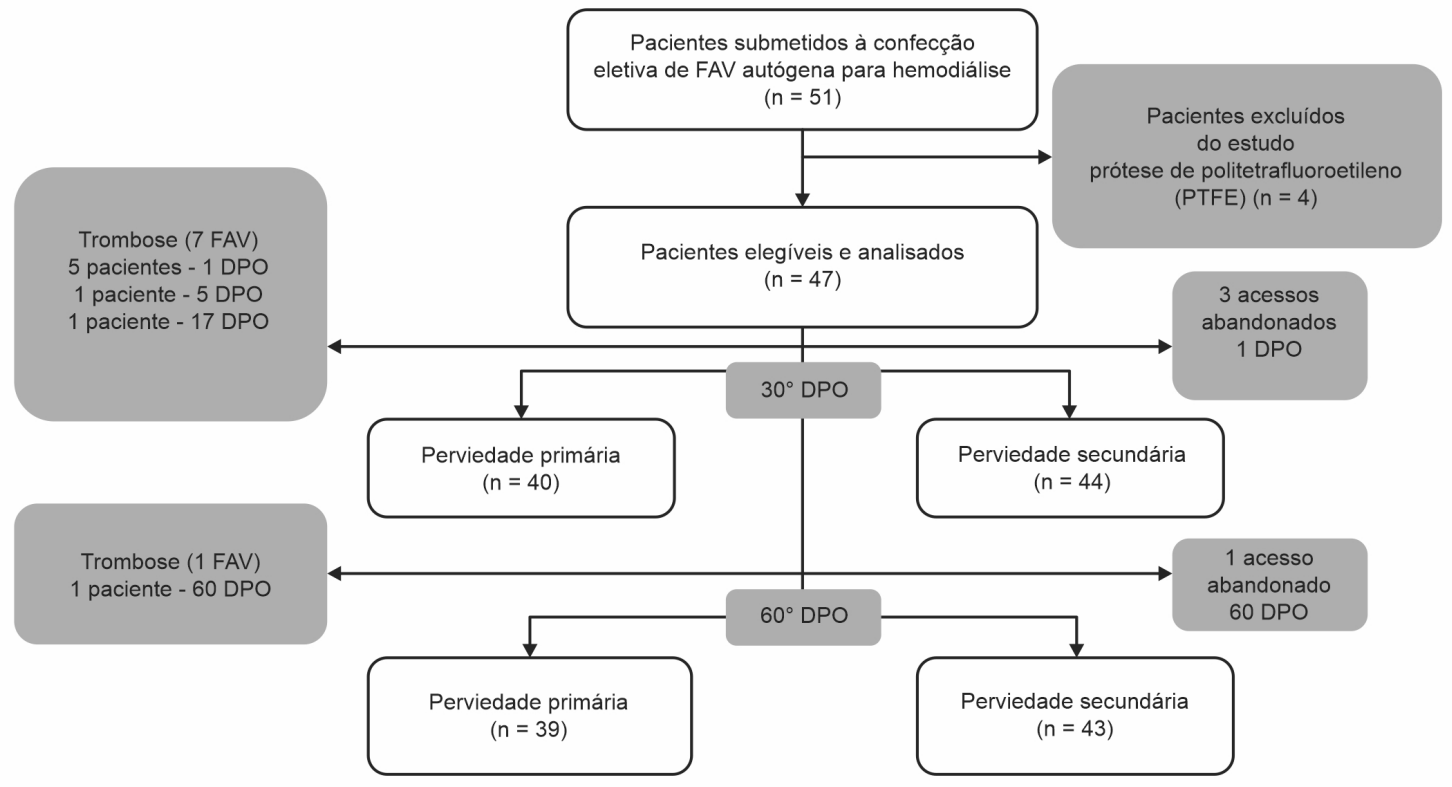

Figura 1. Diagrama de fluxo representando o desenho do estudo. Acesso abandonado é aquele através do qual não é possível mais a realização de diálise. Quando ocorre, é necessário a confecção de um novo acesso. DPO = dias de pós-operatório. 
proximal; e dois pacientes não foram reabordados. Um paciente apresentou oclusão no $4^{\circ}$ dia do pósoperatório e foi submetido a tromboembolectomia com cateter de Fogarty, seguido por reconfecção da anastomose e anticoagulação sistêmica com heparina não fracionada e varfarina. Esse paciente apresentou dois episódios de hematoma de ferida operatória, com necessidade de drenagem cirúrgica, sendo optado por suspensão da anticoagulação sistêmica. No $17^{\circ}$ dia do pós-operatório, outro paciente apresentou trombose da FAV, necessitando de tromboembolectomia com cateter de Fogarty, seguido por reconfecção da anastomose e anticoagulação sistêmica com heparina não fracionada e varfarina. No $60^{\circ}$ dia do pós-operatório, outro paciente apresentou oclusão da FAV após acidente de punção e não foi reabordado (Figura 1).

Ao comparar a VPS e o VF arterial e venoso no intraoperatório, observa-se um maior valor para as

Tabela 1. Variáveis individuais, comorbidades, exames pré-operatórios laboratoriais e de imagem.

\begin{tabular}{|c|c|c|}
\hline \multicolumn{2}{|l|}{ Idade (média \pm DP) } & $56 \pm 15,8$ \\
\hline \multicolumn{2}{|l|}{ Sexo masculino (\%) } & $22(46)$ \\
\hline \multicolumn{2}{|l|}{ IMC (média \pm DP) } & $26,4 \pm 5,9$ \\
\hline \multicolumn{2}{|l|}{ Diabetes (\%) } & $19(40)$ \\
\hline \multicolumn{2}{|l|}{ Hipertensão (\%) } & $40(85)$ \\
\hline \multicolumn{2}{|l|}{ Pré-dialítico (\%) } & $33(70)$ \\
\hline \multicolumn{2}{|c|}{ Bloqueio de plexo braquial (\%) } & $38(81)$ \\
\hline \multicolumn{2}{|c|}{ Hemoglobina (média \pm DP) } & $11,3 \pm 1,75$ \\
\hline \multicolumn{2}{|c|}{ Ureia (média $\pm \mathrm{DP}$ ) } & $120 \pm 37$ \\
\hline \multicolumn{2}{|c|}{$\begin{array}{l}\text { Creatinina mediana (intervalo } \\
\text { interquartil) }\end{array}$} & $4,43(3,2-6,7)$ \\
\hline \multicolumn{2}{|c|}{$\begin{array}{l}\text { Plaquetas - média } \\
\text { (mínimo-máximo) }\end{array}$} & $00(187.000-272.000)$ \\
\hline \multicolumn{2}{|c|}{ RNI (média $\pm \mathrm{DP}$ ) } & $0,96 \pm 0,05$ \\
\hline \multicolumn{2}{|c|}{$\begin{array}{l}\text { Diâmetro artéria doadora } \\
\text { (média } \pm \text { DP) }\end{array}$} & $3,88 \pm 1,40$ \\
\hline \multicolumn{2}{|c|}{$\begin{array}{l}\text { Diâmetro veia receptora } \\
\text { (média } \pm \mathrm{DP} \text { ) }\end{array}$} & $3,57 \pm 1,36$ \\
\hline \multicolumn{3}{|c|}{$\begin{array}{l}\mathrm{DP}=\text { desvio padrão; } I M C \text { = índice de massa corporal; } \mathrm{RNI}=\text { Razão Normalizada } \\
\text { Internacional. }\end{array}$} \\
\hline \multicolumn{3}{|c|}{$\begin{array}{l}\text { Tabela 2. Tipos de fístula arteriovenosa e número de cirurgia } \\
\text { prévias. }\end{array}$} \\
\hline \multirow[t]{7}{*}{ Tipos de FAVs (\%) } & Radiocefálica & $17(36,17)$ \\
\hline & Braquiobasílica & $9(19,15)$ \\
\hline & Braquiobraquial & $2(4,25)$ \\
\hline & Braquiocefálica & $16(34,04)$ \\
\hline & Ulnar-basílica & $1(2,13)$ \\
\hline & Ulno-ulnar & $1(2,13)$ \\
\hline & Radiobasílica & $1(2,13)$ \\
\hline \multirow[t]{4}{*}{ Fístulas prévias (\%) } & 0 & $66(31)$ \\
\hline & 1 & $17(8)$ \\
\hline & 2 & $15(7)$ \\
\hline & 3 & $2(1)$ \\
\hline
\end{tabular}

fístulas funcionantes tanto para perviedade primária quanto secundária comparadas às fístulas não funcionantes (Figura 3). Ao comparar a VPS e o VF arterial e venoso no intraoperatório entre os grupos que apresentaram FAV pérvia e não aos 60 dias, observa-se um maior valor para perviedade primária, mas não para secundária (Tabela 3 ).

A partir desses resultados, foram construídos modelos de predição das perviedades primária e secundária no $30^{\circ}$ dia após a confecção da FAV e da perviedade primária no $60^{\circ}$ dia, de acordo com as variáveis hemodinâmicas. Entre as curvas ROC para perviedade primária no $30^{\circ}$ dia do pós-operatório, o VF arterial mostrou-se como a variável com maior valor preditor para esse desfecho (area under the curve $[\mathrm{AUC}]=0,8095)$ (Figura 4). Os pontos de corte com maior sensibilidade e especificidade para detectar FAVs funcionantes com perviedade primária no $30^{\circ}$ dia do pós-operatório foram: $105,5 \mathrm{~cm} / \mathrm{s}$ para VPS arterial, com sensibilidade de 72,5\% (intervalo de confiança de 95\% [IC95\%]: 57, 17-83,89\%) e especificidade de 71,4\% (IC95\%: 35,89-94,92\%); $106 \mathrm{~cm} / \mathrm{s}$ para VPS venosa, com sensibilidade de 75\% (IC95\%: 59,81-85,81\%) e especificidade de 71,4\% (IC95\%: 35,89-94,92\%);

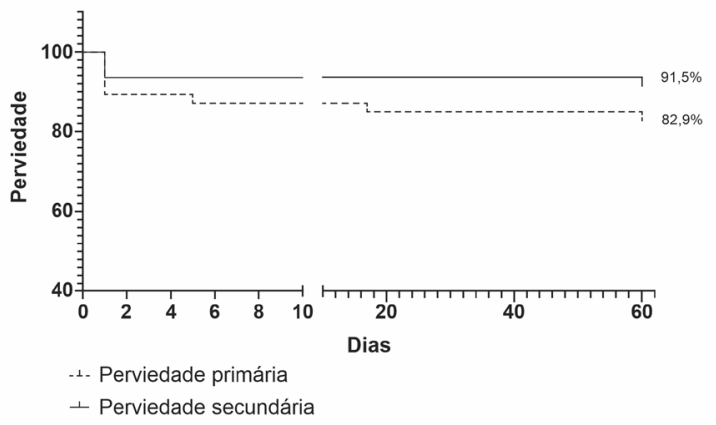

Figura 2. Perviedade primária e secundária das fístulas arteriovenosas para hemodiálise até os 60 dias de pós-operatório.

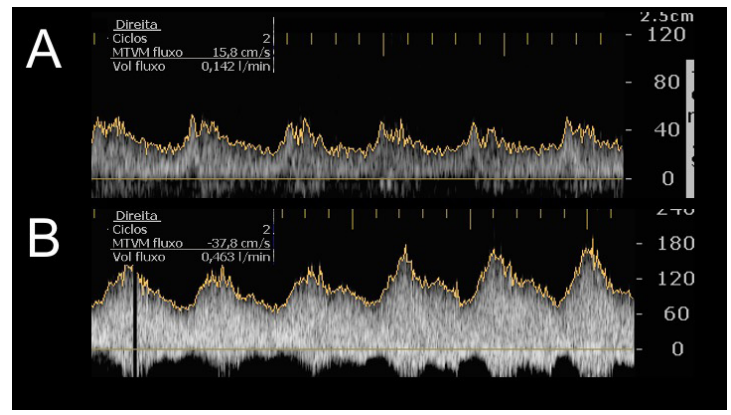

Figura 3. Análise espectral da veia receptora em intraoperatório logo após a confecção da anastomose. 2A) Fístula não funcionante no $1^{\circ}$ dia de pós-operatório. Velocidade sistólica de pico (VPS) 56 $\mathrm{cm} / \mathrm{s}$, volume de fluxo de $142 \mathrm{~mL} / \mathrm{min}$. 2B) Fístula funcionante no $1^{\circ}$ dia de pós-operatório. VPS $192 \mathrm{~cm} / \mathrm{s}$, volume de fluxo de $463 \mathrm{~mL} / \mathrm{min}$. 
Tabela 3. Comparação entre as velocidades de pico sistólico (VPS) e os volumes de fluxo (VF) arteriais e venosos ao ultrassom vascular com Doppler intraoperatório entre as FAV pérvias e não pérvias aos 30 e 60 dias pós-operatórios.

\begin{tabular}{|c|c|c|c|}
\hline \multicolumn{4}{|c|}{ Perviedade primária ao $30^{\circ}$ dia pós-operatório. Valores apresentados em média e desvio padrão } \\
\hline & $\operatorname{Sim}(n=40)$ & Não $(n=7)$ & $\mathbf{p}$ \\
\hline VPS artéria $\mathrm{cm} / \mathrm{s}$ & $150(70,08)$ & $82,93(33,66)$ & 0,0082 \\
\hline VPS veia $\mathrm{cm} / \mathrm{s}$ & $170(80,72)$ & $93,66(46,86)$ & 0,0038 \\
\hline VF artéria $\mathrm{mL} / \mathrm{min}$ & $634(444)$ & $266(261)$ & 0,0084 \\
\hline $\mathrm{VF}$ veia $\mathrm{mL} / \mathrm{min}$ & $602(462)$ & $432(272)$ & 0,4451 \\
\hline \multicolumn{4}{|c|}{ Perviedade secundária ao $30^{\circ}$ dia pós-operatório. Valores apresentados em média e desvio padrão } \\
\hline & $\operatorname{Sim}(n=44)$ & Não $(n=3)$ & $\mathbf{p}$ \\
\hline VPS artéria $\mathrm{cm} / \mathrm{s}$ & $144,4(69,47)$ & $72,3(37,26)$ & 0,0498 \\
\hline VPS veia $\mathrm{cm} / \mathrm{s}$ & $165,5(78,8)$ & $58,47(37,69)$ & 0,0104 \\
\hline VF artéria $\mathrm{mL} / \mathrm{min}$ & $605,7(439)$ & $156,3(100,1)$ & 0,0132 \\
\hline VF veia $\mathrm{mL} / \mathrm{min}$ & $557,6(330,8)$ & $147,3(78,14)$ & 0,0028 \\
\hline \multicolumn{4}{|c|}{ Perviedade primária ao $60^{\circ}$ dia pós-operatório. Valores apresentados em média e desvio padrão } \\
\hline & $\operatorname{Sim}(n=39)$ & Não $(n=8)$ & $\mathbf{p}$ \\
\hline VPS artéria $\mathrm{cm} / \mathrm{s}$ & $150,2(70,92)$ & $88,79(35,30)$ & 0,0157 \\
\hline VPS veia $\mathrm{cm} / \mathrm{s}$ & $170,3(81,76)$ & $102,(49,49)$ & 0,0065 \\
\hline VF artéria $\mathrm{mL} / \mathrm{min}$ & $629,5(449,6)$ & $333,3(308,1)$ & 0,042 \\
\hline VF veia $\mathrm{mL} / \mathrm{min}$ & $552,5(323,7)$ & $428,5(397,9)$ & 0,1185 \\
\hline \multicolumn{4}{|c|}{ Perviedade secundária ao $60^{\circ}$ dia pós-operatório. Valores apresentados em média e desvio padrão } \\
\hline & $\operatorname{Sim}(n=43)$ & Não $(n=4)$ & $\mathbf{p}$ \\
\hline VPS artéria $\mathrm{cm} / \mathrm{s}$ & $144,7(70,26)$ & $86,68(41,86)$ & 0,1058 \\
\hline VPS veia $\mathrm{cm} / \mathrm{s}$ & $165,6(79,73)$ & $84,10(59,8)$ & 0,0508 \\
\hline $\mathrm{VF}$ artéria $\mathrm{mL} / \mathrm{min}$ & $600,6(443,8)$ & $318,3(334,0)$ & 0,1411 \\
\hline VF veia $\mathrm{mL} / \mathrm{min}$ & $394,8(498,9)$ & $544,1(322,3)$ & 0,146 \\
\hline
\end{tabular}

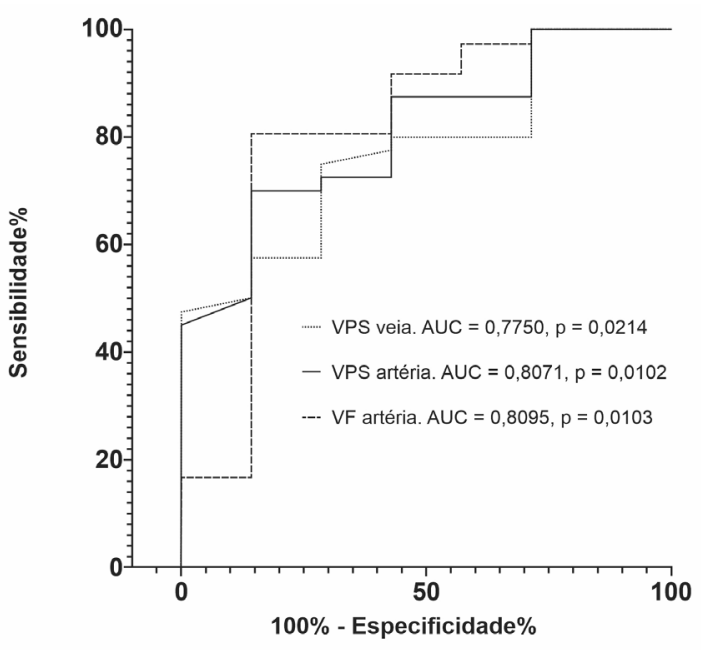

Figura 4. Curvas receiver operating characteristic (ROC) comparando as variáveis ao ultrassom vascular com Doppler em intraoperatório e a perviedade primária aos 30 dias. VPS = velocidade sistólica de pico; VF = volume de fluxo; $\mathrm{AUC}=$ area under the curve. e $290,5 \mathrm{~mL} / \mathrm{min}$ para VF arterial, com sensibilidade de $80,6 \%$ (IC95\%: 64,97-90,25\%) e especificidade de $85,7 \%$ (IC95\%: 48,69-99,27\%) (Tabela 4).

Quanto à perviedade secundária 30 dias após a confecção da FAV, a variável com maior valor preditivo foi o VF venoso (AUC $=0,9545)$ (Figura 5). Para prever esse desfecho, os pontos de corte com maior sensibilidade e especificidade foram: $106 \mathrm{~cm} / \mathrm{s}$ para VPS arterial, com sensibilidade de 72,7\% (IC95\%: $58,15-83,65 \%$ ) e especificidade de 100\% (IC95\%: 43,85-100\%); $230 \mathrm{~mL} / \mathrm{min}$ para VF venoso, com sensibilidade de 86,4\% (IC95\%: 73,29-93,60\%) e especificidade de 100\% (IC95\%: 43,85-100\%); e $262,5 \mathrm{~mL} / \mathrm{min}$ para VF arterial, com sensibilidade de 77,5\% (IC95\%: 62,5-87,68\%) e especificidade de 100\% (IC95\%: 43,85-100\%) (Tabela 4).

Para a perviedade primária no $60^{\circ}$ dia do pósoperatório, o VPS arterial, o VPS venoso e o VF arterial apresentaram AUC de 0,7692, 0,7356 e 0,7321, respectivamente (Figura 6). Os pontos de corte para os maiores valores de sensibilidade e especificidade para esse desfecho foram: $105,5 \mathrm{~cm} / \mathrm{s}$ 
Tabela 4. Sensibilidade e especificidade de diversos pontos de corte para velocidade de pico sistólico (VPS) e volume de fluxo (VF) arterial e venoso ao ultrassom vascular com Doppler intraoperatório para detectar perviedade primária e secundária aos 30 dias do pós-operatório e perviedade primária aos 60 dias do pós-operatório.

\begin{tabular}{|c|c|c|c|c|c|}
\hline \multicolumn{6}{|c|}{ Perviedade primária 30 dias } \\
\hline & Valor & Sensibilidade & IC95\% & Especificidade & IC95\% \\
\hline \multirow[t]{3}{*}{ VPS artéria $\mathrm{cm} / \mathrm{s}$} & $>99,40$ & 72,5 & $57,17 \%$ a $83,89 \%$ & 57,1 & $25,05 \%$ a $84,18 \%$ \\
\hline & $>105,5$ & 72,5 & $57,17 \%$ a $83,89 \%$ & 71,4 & $35,89 \%$ a $94,92 \%$ \\
\hline & $>108,5$ & 70,0 & $54,57 \%$ a $81,93 \%$ & 71,4 & $35,89 \%$ a $94,92 \%$ \\
\hline \multirow[t]{3}{*}{ VPS veia $\mathrm{cm} / \mathrm{s}$} & $>93,85$ & 80,0 & $65,24 \%$ a $89,50 \%$ & 57,1 & $25,05 \%$ a $84,18 \%$ \\
\hline & $>99,15$ & 77,5 & $62,50 \%$ a $87,68 \%$ & 57,1 & $25,05 \%$ a $84,18 \%$ \\
\hline & $>106,0$ & 75,0 & $59,81 \%$ a $85,81 \%$ & 71,4 & $35,89 \%$ a $94,92 \%$ \\
\hline \multirow[t]{3}{*}{$\mathrm{VF}$ artéria $\mathrm{mL} / \mathrm{min}$} & $>262,5$ & 80,6 & $64,97 \%$ a $90,25 \%$ & 71,4 & $35,89 \%$ a $94,92 \%$ \\
\hline & $>290,5$ & 80,6 & $64,97 \%$ a $90,25 \%$ & 85,7 & $48,69 \%$ a $99,27 \%$ \\
\hline & $>318,5$ & 77,8 & $61,92 \%$ a $88,28 \%$ & 85,7 & $48,69 \%$ a $99,27 \%$ \\
\hline \multicolumn{6}{|c|}{ Perviedade secundária 30 dias } \\
\hline & Valor & Sensibilidade & IC95\% & Especificidade & IC95\% \\
\hline \multirow[t]{3}{*}{ VPS artéria $\mathrm{cm} / \mathrm{s}$} & $>93,85$ & 77,3 & $63,01 \%$ a $87,16 \%$ & 66,7 & $11,85 \%$ a $98,29 \%$ \\
\hline & $>99,15$ & 75,0 & $60,56 \%$ a $85,43 \%$ & 66,7 & $11,85 \%$ a $98,29 \%$ \\
\hline & $>106,0$ & 72,7 & $58,15 \%$ a $83,65 \%$ & 100,0 & $43,85 \%$ a $100,0 \%$ \\
\hline \multirow[t]{3}{*}{ VF veia $\mathrm{mL} / \mathrm{min}$} & $>219,0$ & 88,6 & $76,02 \%$ a $95,05 \%$ & 66,7 & $11,85 \%$ a $98,29 \%$ \\
\hline & $>225,0$ & 86,4 & $73,29 \%$ a $93,60 \%$ & 66,7 & $11,85 \%$ a $98,29 \%$ \\
\hline & $>230,0$ & 86,4 & $73,29 \%$ a $93,60 \%$ & 100,0 & $43,85 \%$ a $100,0 \%$ \\
\hline \multirow[t]{3}{*}{ VF artéria $\mathrm{cm} / \mathrm{s}$} & $>238,5$ & 80,0 & $65,24 \%$ a $89,50 \%$ & 66,7 & $11,85 \%$ a $98,29 \%$ \\
\hline & $>251,5$ & 77,5 & $62,50 \%$ a $87,68 \%$ & 66,7 & $11,85 \%$ a $98,29 \%$ \\
\hline & $>262,5$ & 77,5 & $62,50 \%$ a $87,68 \%$ & 100,0 & $43,85 \%$ a $100,0 \%$ \\
\hline \multicolumn{6}{|c|}{ Perviedade primária 60 dias } \\
\hline & Valor & Sensibilidade & IC95\% & Especificidade & IC95\% \\
\hline \multirow[t]{3}{*}{ VPS artéria $\mathrm{cm} / \mathrm{s}$} & $>99,40$ & 71,8 & $56,22 \%$ a $83,46 \%$ & 50,0 & $21,52 \%$ a $78,48 \%$ \\
\hline & $>105,5$ & 71,8 & $56,22 \%$ a $83,46 \%$ & 62,5 & $30,57 \%$ a $86,32 \%$ \\
\hline & $>108,5$ & 69,2 & $53,58 \%$ a $81,43 \%$ & 62,5 & $30,57 \%$ a $86,32 \%$ \\
\hline \multirow[t]{3}{*}{ VPS veia $\mathrm{cm} / \mathrm{s}$} & $>106,0$ & 74,4 & $58,92 \%$ a $85,43 \%$ & 62,5 & $30,57 \%$ a $86,32 \%$ \\
\hline & $>111,1$ & 71,8 & $56,22 \%$ a $83,46 \%$ & 62,5 & $30,57 \%$ a $86,32 \%$ \\
\hline & $>114,6$ & 69,2 & $53,58 \%$ a $81,43 \%$ & 62,5 & $30,57 \%$ a $86,32 \%$ \\
\hline \multirow[t]{3}{*}{ VF artéria $\mathrm{mL} / \mathrm{min}$} & $>262,5$ & 80,0 & $64,11 \%$ a $89,96 \%$ & 62,5 & $30,57 \%$ a $86,32 \%$ \\
\hline & $>290,5$ & 80,0 & $64,11 \%$ a $89,96 \%$ & 75,0 & $40,93 \%$ a $95,56 \%$ \\
\hline & $>318,5$ & 77,1 & $60,98 \%$ a $87,93 \%$ & 75,0 & $40,93 \%$ a $95,56 \%$ \\
\hline
\end{tabular}

IC95\% = intervalo de confiança de 95\%.

para VPS arterial, com sensibilidade de 71,8\% (IC95\%: $56,22-83,46 \%$ ) e especificidade de $62,5 \%$ (IC95\%: $30,57-86,32 \%), 106 \mathrm{~cm} / \mathrm{s}$ para VPS venoso, com sensibilidade de 74,4\% (IC95\%: 58,92-85,43\%) e especificidade de 62,5\% (IC95\%: 30,57-86,32\%), e $290,5 \mathrm{~mL} / \mathrm{min}$ para VF arterial, com sensibilidade de $80 \%$ (IC95\%: 64,11-89,96\%) e especificidade de 75\% (IC95\%: 40,93-95,56\%).

\section{DISCUSSÃO}

Os parâmetros hemodinâmicos no intraoperatório mostraram-se potenciais preditores da perviedade das FAVs para hemodiálise. O VF arterial apresentou o maior valor preditor para a perviedade primária no $30^{\circ}$ dia, com sensibilidade maior que $80 \%$ para o ponto de corte de $290,5 \mathrm{~mL} / \mathrm{min}$. Já para a perviedade secundária no $30^{\circ}$ dia, o VF venoso foi a variável com maior valor preditor, com sensibilidade maior que $85 \%$ e especificidade de $100 \%$ para o ponto de corte de $230 \mathrm{~mL} / \mathrm{min}$. As VPS arterial e venosa demonstraram poder preditivo na perviedade primária do $60^{\circ}$ dia, com pontos de corte de $105,5 \mathrm{~cm} / \mathrm{s}$ e de $106 \mathrm{~cm} / \mathrm{s}$, respectivamente, e com sensibilidade maior que $70 \%$.

Diversos estudos já demonstraram uma associação entre o VF intraoperatório e os principais desfechos das FAVs. Saucy et al. ${ }^{13}$ avaliaram preditores para a falha na maturação das FAVs radiocefálicas nos primeiros 30 dias após sua confecção, encontrando o VF intraoperatório de $120 \mathrm{~mL} / \mathrm{min}$ como ponto de corte, mas com menor taxa de perviedade $(77,58 \%)$. Cyrek et al. ${ }^{14}$ compararam FAVs radiocefálicas de 


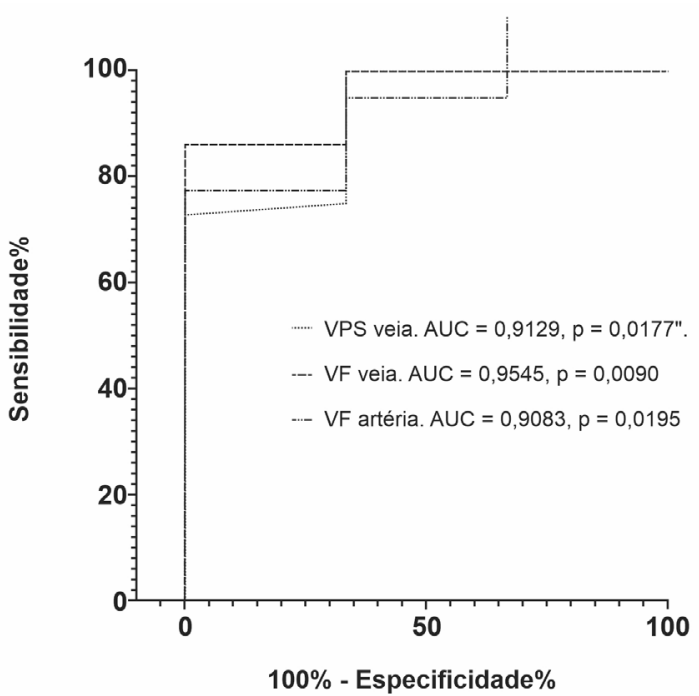

Figura 5. Curvas receiver operating characteristic (ROC) comparando as variáveis ao ultrassom vascular com Doppler em intraoperatório e a perviedade secundária aos 30 dias. VPS = velocidade sistólica de pico; VF = volume de fluxo; $\mathrm{A} \cup \mathrm{C}=$ area under the curve.

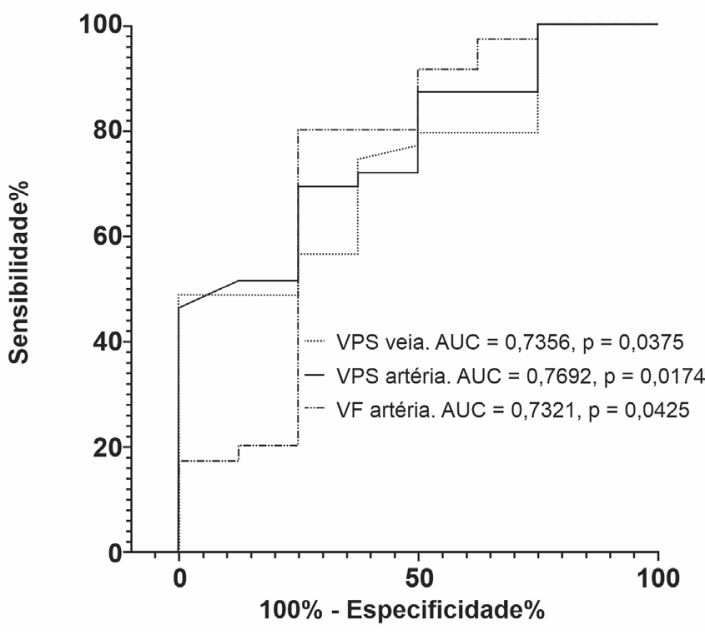

Figura 6. Curvas receiver operating characteristic (ROC) comparando as variáveis ao ultrassom vascular com Doppler em intraoperatório e a perviedade primária aos 60 dias.

alto ( $>200 \mathrm{~mL} / \mathrm{min})$ e baixo $\mathrm{VF}(<200 \mathrm{~mL} / \mathrm{min})$ no intraoperatório e observaram taxas de perviedade primária e secundária significativamente mais altas nas FAVs de alto fluxo ( $100 \%$ e $93,15 \%$, respectivamente) quando comparadas às de baixo fluxo $(81,25 \% \mathrm{e}$ $75 \%$, respectivamente) em 1 ano. Outros estudos observacionais reiteram essa mesma relação entre o VF pós-operatório e a falha da FAV, encontrando pontos de corte que variam entre $160 \mathrm{~mL} / \mathrm{min} \mathrm{e}$ $300 \mathrm{~mL} / \mathrm{min}^{15,16}$.
Em relação aos valores de VPS, diversos estudos corroboram com a associação encontrada. Karanan et al. ${ }^{17}$ analisaram o desfecho das FAVs de acordo com o valor da VPS arterial nos dias 1 e 8 do pós-operatório, confirmando a VPS como preditor do desfecho da FAV de forma significativa. Em concomitância, Abreu et al. ${ }^{18}$ conduziram um estudo com acompanhamento de 5 anos que demonstrou VPS em artérias ulnar e radial como valor preditor para perviedade secundária.

Observamos algumas limitações neste estudo. Tratou-se de estudo em centro único, com único observador, não sendo validadas ou avaliadas as variações e vieses interobservadores. A amostra foi bastante heterogênea, com uma grande variedade de tipos de FAVs. Foram utilizados dois tipos diferentes de aparelhos de ultrassonografia. O aparelho Philips CX 50 foi utilizado para o exame intraoperatório, e o Toshiba Aplio 300, para os exames pós-operatórios. Pode haver uma pequena variação nos valores de VPS e VF entre os aparelhos. Neste estudo, apresentou-se um reduzido número de pacientes; dessa forma, optou-se pela análise univariada, a qual pode apresentar algumas limitações, sobretudo quanto à possibilidade de predição de determinado desfecho. Outra limitação importante foi pequeno número de eventos de não perviedade primária no dia 30 , o que pode ter contribuído para ICs muito amplos.

$\mathrm{O}$ tema abordado nesta pesquisa permanece em aberto e ainda pouco estudado. Por se tratar de propedêutica não invasiva, de ampla distribuição e acesso, com ausência de efeitos colaterais e de riscos para o paciente, a ultrassonografia vascular com Doppler realizada em intraoperatório é uma importante ferramenta para o cirurgião vascular. Esse exame fornece variáveis importantes e em tempo real, as quais podem se correlacionar com o sucesso a curto prazo da cirurgia e modificar a estratégia cirúrgica no intraoperatório - como uma reconfecção do acesso ou até mesmo mudança de local da anastomose - a depender dos valores de VPS e VF. A VPS, apesar de apresentar menor sensibilidade e especificidade quando comparada ao VF, é uma variável de fácil execução e análise, disponível em quase todos os aparelhos de ultrassonografia vascular.

\section{CONCLUSÕES}

A VPS e o VF ao ultrassom vascular com Doppler intraoperatório são preditores de perviedade precoce na cirurgia de FAV para hemodiálise. Ambos representam parâmetros de avaliação confiável para predição da falência precoce da FAV e oferecem a possibilidade de mudança da estratégia cirúrgica no intraoperatório para atingir melhores desfechos no pós-operatório.

\section{REFERÊNCIAS}

1. GBD Chronic Kidney Disease Collaboration. Global, regional, and national burden of chronic kidney disease, 1990-2017: a 
systematic analysis for the Global Burden of Disease Study 2017. Lancet. 2020;395(10225):709-33. PMid:32061315.

2. Carney EF. The impact of chronic kidney disease on global health Nat Rev Nephrol. 2020;16(5):251. http://dx.doi.org/10.1038/ s41581-020-0268-7. PMid:32144399.

3. Liyanage $T$, Ninomiya $T$, Jha $V$, et al. Worldwide access to treatment for end-stage kidney disease: a systematic review. Lancet. 2015;385(9981):1975-82. http://dx.doi.org/10.1016/ S0140-6736(14)61601-9. PMid:25777665.

4. Lok CE, Huber TS, Lee T, et al. KDOQI clinical practice guideline for vascular access: 2019 update. Am J Kidney Dis. 2020;75(4,Suppl 2):S1S164. http://dx.doi.org/10.1053/j.jkkd.2019.12.001. PMid:32778223.

5. Huijbregts HJT, Bots ML, Wittens CHA, Schrama YC, Moll FL, Blankestijn PJ. Hemodialysis arteriovenous fistula patency revisited: results of a prospective, multicenter initiative. Clin J Am Soc Nephrol. 2008;3(3):7149. http://dx.doi.org/10.2215/CJN.02950707. PMid:18256379.

6. Lok CE, Sontrop JM, Tomlinson G, et al. Cumulative patency of contemporary fistulas versus grafts (2000-2010). Clin J Am Soc Nephrol. 2013;8(5):810-8. http://dx.doi.org/10.2215/CJN.00730112. PMid:23371955.

7. Franco RP. Arteriovenous fistulas in hemodialysis: factors of success and the role of nephrologists. J Bras Nefrol. 2018;40(4):309-11. http:// dx.doi.org/10.1590/2175-8239-jbn-2018-0161. PMid:30625232.

8. Bylsma LC, Gage SM, Reichert H, DahI SLM, Lawson JH. Arteriovenous fistulae for haemodialysis: a systematic review and meta-analysis of efficacy and safety outcomes. Eur J Vasc Endovasc Surg. 2017;54(4):513-22. http://dx.doi.org/10.1016/j.ejvs.2017.06.024. PMid:28843984.

9. Hui SH, Folsom R, Killewich LA, Michalek JE, Davies MG, Pounds $\mathrm{LL}$. A comparison of preoperative and intraoperative vein mapping sizes for arteriovenous fistula creation. J Vasc Surg. 2018;67(6):181320. http://dx.doi.org/10.1016/j.jvs.2017.10.067. PMid:29452835.

10. Farber A, Imrey PB, Huber TS, et al. Multiple preoperative and intraoperative factors predict early fistula thrombosis in the Hemodialysis Fistula Maturation Study. J Vasc Surg. 2016;63(1):16370.E6. http://dx.doi.org/10.1016/j.jvs.2015.07.086. PMid:26718822.

11. Castro-Santos G, Salles AG, Dos Anjos GS, Procópio RJ, Navarro TP. Brachial vein transposition: an alternative to hemodialysis arteriovenous graft. J Vasc Bras. 2019;18:e20190077. PMid:31807129.

12. Bazan HA, Schanzer H. Transposition of the brachial vein: a new source for autologous arteriovenous fistulas. J Vasc Surg. 2004;40(1):184-6. http://dx.doi.org/10.1016/j.jvs.2004.03.044. PMid:15218484.

13. Saucy F, Haesler E, Haller C, Déglise S, Teta D, Corpataux J. M. Is intra-operative blood flow predictive for early failure of radiocephalic arteriovenous fistula? Nephrol Dial Transplant. 2010;25(3):862-7. http://dx.doi.org/10.1093/ndt/gfp577. PMid:19892754.

14. Cyrek AE, Bernheim J, Juntermanns B, Husen P, Pacha A, Hoffmann $J N$. Intraoperative use of transit time flow measurement improves patency of newly created radiocephalic arteriovenous fistulas in patients requiring hemodialysis. J Vasc Access. 2020;21(6):990-6. http://dx.doi.org/10.1177/1129729820916561. PMid:32375582.

15. Benaragama KS, Barwell J, Lord C, et al. Post-operative arteriovenous fistula blood flow influences primary and secondary patency following access surgery. J Ren Care. 2018;44(3):134-41. http://dx.doi.org/10.1111/jorc.12238. PMid:29520968.

16. Usta E, Elkrinawi R, Salehi-Gilani S, et al. Risk factors predicting the successful function and use of autogenous arteriovenous fistulae for hemodialysis. Thorac Cardiovasc Surg. 2013;61(5):438-44. PMid:23169107.

17. Karanam S, Dasari R, Kumar A, Tyagi A, Kumar S, Lakshmi AY. Hemodialysis arteriovenous fistula maturation and role of perioperative vascular mapping. J Dr NTR Univ Health Sci. 2019;8(4):257-60. http://dx.doi.org/10.4103/JDRNTRUHS.JDRNTRUHS_55_19.

18. Abreu R, Rioja S, Vallespin J, Vinuesa X, Iglesias R, Ibeas J. Predictors of early failure and secondary patency in native arteriovenous fistulas for hemodialysis. Int Angiol. 2018;37(4):310-4. http:// dx.doi.org/10.23736/S0392-9590.18.03927-5. PMid:29424185.

Correspondência Guilherme de Castro Santos Universidade Federal de Minas Gerais - UFMG Rua Professor Arduíno Bolivar, 276, apartamento 500, Bairro Santo Antônio

CEP: 30350-140 - Belo Horizonte (MG), Brasil Tel.: (31) 3409-9759 E-mail: gcs2000@gmail.com

Informações sobre os autores

GCS - Cirurgião Vascular, Sócio Efetivo da Sociedade Brasileira de Angiologia e Cirurgia Vascular (SBACV); Professor Assistente, Departamento de Cirurgia, Faculdade de Medicina, Universidade Federal de Minas Gerais (UFMG); Mestre em Cirurgia; Doutorando em Cirurgia, UFMG. GYS - Acadêmica de Medicina, Voluntária do Programa de Iniciação Científica, Universidade Federal de Minas Gerais (UFMG). RMSO - Acadêmica de Medicina, Bolsista de Iniciação Científica pelo Programa da ADRC, Pró-reitoria de Pesquisa da Universidade Federal de Minas Gerais (UFMG).

RJP - Cirurgião Vascular e Endovascular, Sócio Efetivo da Sociedade Brasileira de Angiologia e Cirurgia Vascular (SBACV), Coordenador do Serviço de Cirurgia Endovascular, Hospital das Clínicas, Universidade Federal de Minas Gerais (UFMG).

TPN - Cirurgião Vascular e Endovascular, Sócio Titular da Sociedade Brasileira de Angiologia e Cirurgia Vascular (SBACV); Professor

Associado, Departamento de Cirurgia, Faculdade de Medicina, Universidade Federal de Minas Gerais (UFMG); Doutor em Cirurgia pela UFMG; Coordenador do Serviço de Cirurgia Vascular, Hospital das Clínicas, UFMG.

Contribuição dos autores Concepção e desenho do estudo: GCS, TPN Análise e interpretação dos dados: GCS Coleta de dados: GCS, GYS, RMSO Redação do artigo: GCS, GYS, RMSO Revisão crítica do texto: RJP, TPN Aprovação final do artigo*: GCS, GYS, RMSO, RJP, TPN Análise estatística: GCS Responsabilidade geral pelo estudo: GCS, TPN

*Todos os autores leram e aprovaram a versão final submetida ao J Vasc Bras. 